Cuestiones de Sociología, nº 18, e050, junio 2018, ISSN 2346-8904

Universidad Nacional de La Plata

Facultad de Humanidades y Ciencias de la Educación

Departamento de Sociología

\title{
Deporte y modernidad en Argentina: problemas conceptuales y propuesta de abordaje
}

\author{
Sazbón, Daniel \\ Universidad de Buenos Aires - Universidad Nacional de La Plata - Universidad Nacional \\ Arturo Jauretche, Argentina \\ dsazbon@gmail.com \\ Frydenberg, Julio \\ Universidad Nacional de San Martín - Universidad Nacional de La Plata, \\ Argentina jufryde@gmail.com
}

Cita sugerida: Sazbón, D. y Frydenberg, J.(2018). Deporte y modernidad en Argentina: problemas conceptuales y propuesta de abordaje. Cuestiones de Sociología, 18, e050. https://doi.org/10.24215/23468904e050

(1)(2) Esta obra está bajo licencia Creative Commons Atribución-NoComercial-CompartirIgual 4.0 Internacional BY No $\mathrm{sA}$ http://creativecommons.org/licenses/by-nc-sa/4.0/deed.es_AR 


\section{Deporte y modernidad en Argentina: problemas conceptuales y propuesta de abordaje}

Sports and modernity in Argentina: conceptual problems and approach proposal

Daniel Sazbón

Universidad de Buenos Aires - Universidad Nacional de La

Plata - Universidad Nacional Arturo Jauretche, Argentina

dsazbon@gmail.com

Julio Frydenberg

Universidad Nacional de San Martin - Universidad

Nacional de La Plata, Argentina

jufryde@gmail.com

\section{Resumen:}

En el campo de historia del deporte es clásica su conceptualización como símbolo de las transformaciones sociales que oponen el modo de vida "tradicional" del "moderno" es de vieja data. Tal identificación entre deporte y "modernidad" es lugar común tanto en los estudios focalizados en su contexto europeo (especialmente británico) de origen como en aquellos espacios en los que su difusión planetaria logró imponerse con mayor o menor rapidez, sirviendo como indicador de su incorporación al "moderno" mundo capitalista.

El término modernidad resulta en nuestra opinión problemáticopara estos propósitos, tanto por la laxitud con la que ha sido utilizadocomo por su particular amplitud a la hora de abarcar procesos complejos y contradictorios ocultando la tensión existente entre sus diferentes planos, que terminan resolviéndose en una imagen de engañosa simplicidad. Por lo tanto, el objetivo de nuestro trabajo será señalar las dificultades que presenta la categoría modernidad para dar cuenta de la conexión entre el desarrollo de las actividades deportivas y el proceso de transformaciones vividas por la sociedad argentina -y en general, latinoamericana- en el período comprendido entre fines del siglo XIX y comienzos del XX.

Palabras claVe: Modernidad, Deporte, Argentina, América Latina, Siglo XIX.

\section{ABstract:}

In the field of sports history, its conceptualization as a symbol of the social transformations that oppose the "traditional" to the "modern" way of life is typical. Such identification between sport and "modernity" is commonplace both in the studies focused on its European (and particularly British) context of origin as in those spaces in which its planetary diffusion led to its incorporation, serving as an indicator of its incorporation to the "modern" capitalist world.

In our view the term modernity results problematic for these purposes, as much because of the laxity with which it has been used as due to its particular broadness, encompassing complex and contradictory processes and hiding the tension existing between its different planes, resolving them in deceitfully simple image. Therefore, the objective of our work will be to point out the difficulties presented by the category modernity to account for the connection between the development of sports activities and the process of transformations experienced by Argentine -and generally Latin American- societies in the period between the end of the 19th century and the beginning of the 20th.

KEYWORDS: Modernity, Sport, Argentina, Latin America, XIXth Century.

En el campo de historia del deporte, la conceptualización que se hace de él como símbolo de las transformaciones sociales en la transición entre el modo de vida "tradicional" y el por contraste llamado "moderno" es de vieja data. Este hecho es particularmente apreciable en la historiografía británica, en la cual el desarrollo del sport es visto como inseparable del contexto más amplio de cambios dentro de la transición hacia la sociedad "burguesa", siendo el deporte tanto expresión de esos cambios como instrumento que permite su realización, así como su despliegue a escala universal. Del mismo modo, en las regiones en las que el deporte británico logró imponerse con cierto éxito - tanto en el resto de Europa como en zonas más alejadas-, 
es común encontrar una similar aproximación al estudio de estas actividades a partir de la utilización de las categorías que opondrían la sociedad "antigua" a la "moderna", simbolizada por la incorporación de estas prácticas novedosas (por ejemplo el tratamiento del cricket en Appadurai, 2001); en este sentido, el caso latinoamericano no es la excepción.

No obstante, si bien esta vinculación entre las formas específicas de las actividades deportivas que se desarrollan en la Inglaterra de los siglos XVIII-XIX y el conjunto de atributos que distinguen a las sociedades actuales de las precedentes son un tópico habitual del estudio del deporte, estos abordajes no siempre comparten una misma forma de concebir estos atributos, la relación que guardarían entre sí, o la forma en que estarían ligados al mundo del deporte. El privilegio dado a alguno de los múltiples planos que componen el paisaje del mundo actual (económico, cultural, político, social, etc.), en este sentido, supone una divergencia en cuanto al enfoque que se haga de las prácticas deportivas, ya sea como resultantes de estos cambios o bien como agentes del mismo.

El empleo de la categoría modernidad, en este sentido, ha permitido que en muchos casos esta dificultad no salte a la vista, oculta tras el manto común que proporciona el término a los numerosos y diversos aspectos de la sociedad contemporánea que pueden ser considerados relevantes en los análisis de la misma. Por otro lado, las características del concepto contribuyen a que no sea sencillo precisar su uso, por lo que la pregunta acerca de las relaciones entre el deporte y la "modernidad" específica de un caso nacional, como puede ser el argentino, no tiene una respuesta unívoca a menos que se delimite el sentido del término.

Nuestro objetivo en este trabajo es señalar algunos de los inconvenientes que acarrea el uso mecánico e indiscriminado de categorías como modernidad para el análisis histórico de procesos como el del desarrollo de las formas actuales que adoptan las competencias deportivas. Partimos de la idea de que entre ambos planos -el de la "modernización" y de la "deportivización" de las prácticas- existen lazos evidentes, pero también puntos de roce y hasta de contradicción; esto se debe a que fenómenos culturales con un cierto grado de complejidad, como en este caso ocurre con el deporte, cargan en su interior no sólo los atributos y rasgos propios de la sociedad de su tiempo, como ocurriría en este caso con la pujante sociedad capitalista del siglo XIX, sino también tradiciones, permanencias y continuidades pertenecientes a formas mentales y materiales mucho más antiguas. No pretendemos negar de plano la asociación entre "deporte" y "modernidad" (o entre "deporte" y "capitalismo", aunque aquí existen derivas posibles hacia las que no nos dirigiremos en este trabajo), sino mostrar la inadecuación del término para agotar con él una lectura que dé cuenta de la riqueza y complejidad del fenómeno deportivo.

Es por tal motivo que antes de comentar la vinculación entre deporte y "modernidad" en Argentina presentaremos unas breves líneas acerca de las dificultades que presenta en nuestra opinión esta categoría. Desde luego, las páginas que siguen deben ser leídas como un intento de problematización que no pretende cerrar la discusión, sino que por el contrario supone dejarla abierta, entendiendo que se trata de un debate necesario para el fortalecimiento del campo de estudios del deporte. Tampoco se deben comprender estos apuntes como una requisitoria contra el empleo de modelos y herramientas provenientes de disciplinas y abordajes diversos -sociológicos, económicos, culturales, antropológicos, etc. - y a favor de una improbable historia meramente "empírica", basada en la recopilación y ordenamiento de datos y reactiva a su absorción por conceptos o categorías "teóricas"; como ya ha sido argumentado ampliamente, consideramos que la labor del historiador resulta imposible sin el empleo de las herramientas conceptuales que les permitan "ordenar el caos de los datos observables en objetos comprensibles para la mente humana” (Weber, 1997).

\section{LA TRADICIÓN DE LA “MODERNIDAD”}

Pocos términos han sufrido más abusos que el de moderno y sus asociados, modernidad, modernización, etc.; pocos son más difíciles de precisar en su sentido último. Refiriendo esencialmente, desde su raíz, a una ruptura de orden temporal -que opondría la actualidad de la moda al uso de las costumbres anteriores- (Koselleck, 
1993), su aplicación en los diversos campos del conocimiento que han echado mano de ellos es amplísima, lo que los ha hecho tan habituales como imprecisos. Tal vaguedad se puede verificar a lo largo de múltiples ejes, que se implican mutuamente, ya que al multiplicarse las referencias posibles de los términos, lo mismo ocurre con sus fronteras delimitadoras. ${ }^{1}$

Ejemplo clásico de las dificultades de la deixis en relación con el vocabulario científico, lo "moderno" no podría ser entendido, por lo tanto, sin referencia a las condiciones precisas de enunciación en que tiene lugar su utilización. De este modo, y de acuerdo al contexto específico al que se quiera referir, el conjunto de atributos epocales a los que se quiere denotar con la referencia a lo "moderno" puede extenderse tanto al siglo XV-XVI de la historia "occidental” (un término para el que caben las mismas precauciones que para el aquí nos ocupa), convencionalmente entendido como inicio de la etapa a la que se denomina, precisamente, Historia Moderna, y que estaría ilustrado con episodios disruptivos como el descubrimiento del continente americano o la invención de la imprenta, como así también a etapas mucho más cercanas, donde condensaría un conjunto de sensibilidades, experiencias, hábitos y formas de relacionarse, consideradas todas "modernas". 2

Las propias dificultades del término explican la paradójica situación de que mientras ciertas conceptualizaciones consideran que su despliegue es aún incompleto -o bien que la propia naturaleza del objeto hace del mismo un desarrollo siempre en curso y nunca agotable-, ya sea en términos generales como movimiento histórico que abarca al conjunto humano, ya sea en cuanto a la aplicación local en contextos específicos del mismo, por otro lado para otras tematizaciones la referencia alude a un período histórico que ya pertenecería al pasado, y con el que nuestra propia configuración actual se contrastaría con nitidez. Así, la modernidad puede ser tanto un "proyecto inconcluso", un despliegue inextinguible, un objetivo aún no alcanzado, un conjunto de rasgos desaparecidos que se evoca con nostalgia, o incluso una ilusión nunca verificada (Latour, 2007).

En todos estos casos, el articulador común que unifica el uso de estas categorías es la referencia a un conjunto de atributos que distinguirían a un determinado momento histórico del anterior, del cual surge y frente al cual se coloca en disonancia. Este aspecto "rupturista" de la "modernidad" puede tomar formas diversas, que implican a su vez ritmos de cambio diversos; esto explica que en algunos casos el concepto se asocie con los procesos de transformación política, económica o cultural de velocidad más o menos intensa, y en los que en muchos casos sus protagonistas asumen consciente y voluntariamente el contenido innovador de tales modificaciones, y en otras instancias se lo vincula con modificaciones que tienen lugar a un ritmo mucho más pausado, y cuyos resultados sólo serán perceptibles tras un largo proceso acumulativo.

Que en ambos casos se pueda haber hecho uso de otra categoría tan polisémica y difícil de precisar unívocamente como revolución no hace más que reflejar la íntima conexión que existe entre estos conjuntos terminológicos. Como han mostrado numerosos autores, de lo que se trata es de una determinada forma de experimentar la temporalidad por parte de los seres humanos; la referencia al cambio histórico es así un componente insustituible de la "experiencia de modernidad"; de allí que, más que categoría cronológica haya que pensar el uso de modernidad en el sentido cualitativo que le han dado la mayor parte de quienes lo emplean (Berman, 1989; sobre "modernidad" y "revolución", Koselleck, 1993).

Al sustantivar en una categoría abarcativa una multiplicidad de atributos de competencia específica (políticos, económicos, sociales, culturales, psicológicos, etc.), el término pasa a denotar una etapa cronológica y cualitativamente específica, ubicada en un momento determinado del desarrollo histórico, y caracterizada precisamente por la demarcación que supone la realización plena de los cambios a los que se refieren las características mencionadas. Al mismo tiempo, entendida como producto de un conjunto de transformaciones que tiene como resultado la quiebra de los rasgos de las etapas anteriores para dar paso a la novedad "moderna", el concepto se puede referir tanto al resultado de esos cambios como a la condición misma del proceso transformador, condición que se pretendería, por su propia naturaleza, siempre inacabada. 
Por otro lado, una deriva se desprende del concepto, por el carácter fuertemente teleológico del movimiento por el cual los "nuevos tiempos" pugnan con los "antiguos" para imponerse -enfrentando la resistencia de las instituciones en los que estos últimos estarían condensados-, lo que supone la existencia de una dirección unívoca del desarrollo histórico; la "modernización" sería la incorporación irremediable de estas novedades, así como la remoción no menos obligada de sus obstáculos. ${ }^{3}$ Este carácter necesario de la modernidad implica asumir la universalidad de un proceso histórico que se aplicaría urbi et orbi, más allá de las características particulares de los contextos. De aquí han surgido críticas al concepto que apuntan tanto a su carácter finalista como a la unidireccionalidad del movimiento; habría así no una sino varias posibles “modernidades" o "modernizaciones" (García Canclini, 2001;Eisenstadt, 2003; Wagner, 1994). ${ }^{4}$

Abstracción y síntesis de transformaciones particulares, sustantivación del conjunto de atributos que conformarían una etapa histórica a la que se define por estas mismas cualidades, homogenización de procesos complejos y muchas veces contradictorios, delimitación cronológica difusa que recorta un período de longitud y entidad variable, coronación de un movimiento que se define tautológicamente por sus mismas características, las limitaciones del término modernidad parecen al mismo tiempo ser una de las causasdel éxito de su empleo. Ha permitido que autores y escuelas de orientaciones muy diversas lo adopten indiscriminadamente, con el peligro de incomprensión mutua que esto conlleva.

\section{MODERNIDADES}

Estas dificultades de precisión pueden explicar las diversas modalidades de utilización de estas categorías por parte de tradiciones académicas y disciplinarias que no sólo han hecho uso del mismo en forma particular, sino que en ocasiones lo hacen sin percibir las dificultades y eventuales contradicciones a las que puede llevar esta diversidad. De todos modos, la propia naturaleza del término y su inmediata referencia a la existencia de algún tipo de modificación cualitativo-cronológica, como hemos dicho, hará que en todos los casos, si bien bajo modalidades distintas, se mantenga el elemento común, constitutivo del uso del mismo: la polaridad que opone a lo moderno un conjunto de atributos contra el cual estese destaca, y que tendrá en cada caso una referencia y un contenido específico.

Mencionemos en primer lugar el uso del término en las obras más cercanas al campo de la filosofía y la historia de las ideas a partir de la preocupación por abarcar los atributos que caracterizarían una etapa histórica particular. Aquí, modernidad suele referir a un conjunto de modificaciones que suelen centrarse en las condiciones en las que tienen lugar la actividad intelectiva: serán "modernas" entonces las posibilidades de conocimiento del ser humano, y al mismo tiempo las condiciones de acceso al saber, así como la relación que se postula entre dicho saber y el estatuto de verdad que posee.

Estas modificaciones que se sintetizan bajo el apelativo de modernidad implican la apertura de un campo del conocimiento anteriormente restringido por obra de instituciones encargadas de su control, el acceso a herramientas y capacidades novedosas que amplían de igual modo tal campo, la dilución de la jerarquía tradicional asociada a estos saberes (y eventualmente, de toda jerarquía en este terreno), etc. En suma, en esta acepción se entendería por modernidad una serie de innovaciones, vinculadas a categorías como secularización, laicismo, racionalismo, etc.; la oposición paradigmática entre "tradición" y "modernidad" sería equivalente a la que suele condensarse en las referencias a la Ilustración, el progreso, etc. El fin de la tutela religiosa y del respeto ciego a las costumbres heredadas corresponde de este modo al nacimiento y desarrollo del sujeto "moderno" de conocimiento, dotado de capacidades de razonamiento autónomo y más o menos independiente del peso del colectivo (Habermas, 1998; Foucault, 2002;Jameson, 2004; en cuanto a las características del sujeto "moderno": Touraine, 1993).

Si en este sentido la "modernidad" implica la apertura de posibilidades de emancipación del género humano, otros acercamientos han alertado sobre los efectos últimos que tendría esta separación del hombre de cualquier referencia última colocada en un plano trascendente: desde esta perspectiva, el rasgo distintivo 
de los tiempos modernos estaría en el avance del conocimiento "práctico" pero sólo a partir de la inevitable ausencia de "sentido" de tal capacidad intelectual, volcada únicamente al "mundo de las cosas". El abandono de su subordinación a una autoridad externa habría liberado así a la Razón a costa de transformarla en mera razón "técnica”, al tiempo que quedaría imposibilitada cualquier justificación "racional" de los principios que deben regir la vida de los hombres: una distancia cada vez mayor se abriría de este modo entre el mundo de los "fines" y el de los "valores" en los tiempos modernos (Weber, 1997). ${ }^{5}$

En un sentido más vinculado a la filosofía política, el uso del término suele denotar un aspecto particular de tal proceso más amplio, el vinculado con los cambios en las concepciones referidas a la legitimidad del poder político, la fuente de la soberanía, el carácter trascendente o inmanente de la autoridad, etc. Acotada a esta dimensión, la irrupción de la "modernidad" implicaría un nuevo modelo de concepción del lazo político que se opondría al vigente tradicionalmente, un AncienRègime cuyo desalojo puede ser más o menos rápido y violento, es decir, más o menos "revolucionario" o progresivo, y que en algunas acepciones puede constituir un proceso aún en curso. Lo que más arriba aparecía desde el punto de vista del "sujeto de conocimiento" es en este sentido leído desde el ángulo concomitante del "sujeto político".

Por otro lado, en el terreno económico, se ha utilizado la referencia a formas de producción "modernas" indistintamente para referirse tanto al sistema de economía de mercado in totu, como a modalidades particulares de su desarrollo, temporalmente situadas dentro de un momento particular de su despliegue histórico. En igual sentido, la utilización del término permite contraponer tal forma específica de producción a modelos anteriores, que oscilan entre la referencia general a cualquier sistema que no pueda referirse como plenamente "capitalista" (economías de subsistencia, o basadas en relaciones personales, o bien escasamente monetarizadas, etc.) y modalidades de "capitalismos" que se consideran pretéritas (mercantiles, industriales, fordistas, "renanas", etc.). Y si anteriormente pensábamos al protagonista y resultado de esta transición en cuanto a su autonomía intelectual y política, aquí se lo tematiza en función de su relación con el mercado: la modernidad de este sujeto, en este sentido, va de la mano de su presencia en el mismo como productor y consumidor de mercancías. ${ }^{6}$

Como se observa, en cada una de estas grandes formas de utilización de la categoría, los tiempos anteriores a los "modernos", con los que estos vienen a recortarse, se pueden también considerar sustantivados a partir de la referencia a un conjunto de instituciones, relaciones sociales, etc., que constituirían por ello mismo algunos de los principales obstáculos para el despliegue de modernidad que implica la transición histórica así concebida: así, la Iglesia, la monarquía y la nobleza feudal aparecen icónicamente como la contracara por excelencia de los nuevos tiempos, así como, en sentido inverso, un indicador más o menos preciso del grado de avance de estos.

En este sentido, y sin que siempre haya un uso explícito de la categoría, la reflexión sociológica clásica(la que tiene lugar entre la segunda mitad del siglo XIX y las primeras décadas del siguiente) encuentra precisamente en esta transición hacia un "nuevo mundo" el objeto privilegiado de un análisis disciplinario que fundamenta su especificidad como campo autónomo. Tanto en la obra de sus "padres fundadores" como en la de buena parte de sus epígonos, la sociología se ha constituido fundamentalmente alrededor de la delimitación entre dos conglomerados conceptuales e históricos más o menos delimitados, el mundo "tradicional" y el "moderno". Las conceptualizaciones con las que se da cuenta de ambos polos varían, desde luego, de acuerdo al eje privilegiado del análisis, pero este elemento diacrónico permanece: Comunidad vs. Sociedad, Feudalismo vs. Capitalismo, Sagrado vs. Secular, Tradicional vs. Racional, Rural vs. Urbano, etc. ${ }^{7}$

Precisamente alrededor de la última de estas polaridades es la que el uso de la categoría se ha dilatado temáticamente en el campo de los llamados “estudios culturales”, quizás el área disciplinaria que con mayor aliento ha utilizado la referencia a la "modernidad" para referirse a las características centrales de los tiempos actuales, así como al carácter más o menos acabado del despliegue histórico de esta etapa en relación con la precedente, y utilizando para ello categorías y herramientas tomadas de disciplinas diversas, particularmente la antropología. Desde esta perspectiva, la tónica de los tiempos “modernos” estaría dada principalmente por 
la contraposición con los ritmos que anteriormente regían la vida en las comunidades tradicionales: la ciudad y su explosiva dinamización de las transformaciones que pueden experimentar sus habitantes, así como el aumento en el número y densidad de las relaciones entre ellos, se constituye de este modo a la vez en escenario y en condensación de la nueva etapa histórica. ${ }^{8}$

Una nueva sensibilidad sería de este modo la característica por excelencia del período "moderno", cuyos tópicos más significativos son la velocidad y multiplicidad de estímulos a los que están expuestos sus miembros; la relativización de las fronteras que separan lo habitual de lo inaudito, reverso de la expansión de los límites de lo que es posible esperar; la consecuente dilución de la consistencia del mundo conocido, de su estabilidad y permanencia; una aceleración del ritmo de los cambios históricos imaginables, y por lo tanto una dilatación de la temporalidad de los mismos; la fragmentación de la personalidad del sujeto, hasta el punto de poner en discusión la misma noción; una disminución en la confianza de las posibilidades de la capacidad intelectiva humana para dar cuenta del proceso en el que se ve envuelto; etc. En definitiva, una nueva y "moderna" forma de la experiencia humana, cruzada fundamentalmente por una radicalización de su temporalidad constitutiva, ante la constatación de que "todo lo sólido se disuelve en el aire" (Berman, 1989; Koselleck, 1993; Rosa, 2005).

De esta forma, la modernidad de un conjunto social o de un período histórico refiere al mismo tiempo a las condiciones sociales que la hacen posible, a las formas en que tales condiciones y tal período son experimentados por sus contemporáneos, y las condiciones mismas de incorporación de esas experiencias. Lo moderno es así tanto el sistema social como las posibilidades de intelección de tal sistema que poseen quienes viven en él, y de las modalidades de relación que se establecen entre ellos: tanto una forma de vida, entonces, como una forma de conciencia, una conciencia de la temporalidad y una "actitud" ante ella, son así subsumidas en la abstracción de ser, fundamentalmente, modernas.

\section{DEPORTE Y MODERNIDAD}

Habiendo señalado de este modo algunos de los que consideramos puntos débiles de la categoría modernidad, la pregunta sobre la vinculación entre el deporte y la sociedad "moderna"-o su equivalente, la de los deportes como agentes de "modernización”- necesita traducirse por otra más precisa: ¿cuáles son los elementos novedosos de los deportes actuales? ¿En qué sentido estas prácticas son subsidiarias del desarrollo de las nuevas formas de vida colectiva? Este es el punto central sobre el que creemos deberían echar luz las investigaciones históricas sobre las actividades deportivas. Nos enfrentamos aquí a una dificultad de segundo orden, ya que a las contradicciones implícitas en conceptos como modernización se les agrega la ambigüedad del lugar de las prácticas deportivas en el funcionamiento de los grupos humanos; no casualmente una de las tematizaciones más atentas a las especificidades de dicho espacio ha preferido utilizar categorías analíticas que enfatizan su condición de "zona libre", dotada de un grado de "liminariedad" en relación a las condiciones estructurales del conjunto social que las ubica al mismo tiempo tanto "dentro" como "fuera" de las mismas. Lo mismo han hecho los abordajes que, desde posiciones teóricas distintas, han coincidido en reconocer la especificidad y relativa autonomía de las prácticas deportivas respecto al conjunto del que forman parte (Archetti, 2003).9

Quien con mayor aliento ha encarado el estudio del deporte desde una perspectiva tributaria de la categoría modernización ha sido el norteamericano Allen Guttmann, cuyo modelo de conceptualización de las prácticas deportivas "modernas" se sostiene en seis ejes, que equivalen a otros tantos atributos de su postulada modernidad: secularismo en la competencia, igualdad de los competidores, especialización de los deportistas, racionalización del sistema de reglas que rige la actividad, burocratización de las instituciones y organismos donde se los practica y, finalmente, énfasis en la cuantificación de los resultados, rasgo que se corporiza en la centralidad del "récord" como símbolo que condensa este nuevo espíritu que anima a las actividades competitivas en nuestras sociedades (Guttmann, 1978). 
Como lo explicita su autor, la referencia a la "modernidad" del deporte abreva aquí en la perspectiva weberiana (en la versión canonizada por Talcott Parsons): ${ }^{10}$ un modelo de relaciones sociales que va de la mano de la adopción de un sistema de regulaciones políticas y de una concepción del empleo de las facultades intelectivas humanas. Si la rigidez del esquema lo habilita para ser utilizado con cierta facilidad para comparar casos puntuales y comprobar su mayor o menor "modernidad", también lo ha hecho objeto de no pocas críticas, particularmente desde los estudios post-coloniales, que objetan la simplificación eurocentrista que reduce a "primitiva" toda forma deportiva alternativa a la considerada por Guttmann "moderna" (Carrington, 2010).

Por otro lado, el corte tajante que supone el modelo guttmanniano entre las formas modernas de las prácticas deportivas y sus predecesoras resulta útil en primera instancia para confeccionar una suerte de "tipo ideal" del deporte moderno, pero precisamente por tal idealización estereotipada implica riesgos a la hora de analizar el material empírico con el que trabajamos los especialistas en el campo. Este peligro es particularmente notorio en la lectura que puede hacerse de la esfera de los valores o "mentalidades" con los que los competidores participan de tales actividades: un elemento crucial de esta esfera como la tan conocida "caballerosidad deportiva" o sportsmanship, de importancia central en las formas que adopta el despliegue de estas prácticas competitivas en el seno de la comunidad británica -tanto en su país de origen como en su expansión planetaria-, no puede ser entendido sin remitir a códigos de conducta que esquemáticamente corresponderían al universo "tradicional” o "pre-moderno" ("espíritu cortés”, "gentlemanship"), y al mismo tiempo intrínsecamente "modernos" (universalidad de las reglas, igualdad entre contrincantes, etc.). ¿Cómo conciliar esta ambigüedad inherente al objeto en los nítidos lineamientos de la "modernidad"?

Este tipo de críticas, nos apresuramos a reconocerlo, no suponen desconocer la evidente vinculación entre el surgimiento de las actividades deportivas en la forma que adoptan hacia los siglos XVIIIXIX y las características del entramado social en el que tiene lugar su aparición; por el contrario, tal vínculo constituye uno de los principales temas de análisis de las investigaciones del campo. Pero ni tal proceso de transformaciones goza de una homogeneidad tal como para condicionar de manera unívoca las modificaciones en todos los campos que conforman el mosaico de la existencia humana, ni de ningún modo tal "vinculación" puede equivaler a una "determinación" que permita subsumir todo cambio en una misma y uniforme "modernización”.

- la especificidad de las asociaciones creadas para la práctica del deporte (clubes, ligas, federaciones), y su tendencia a mantener un elevado grado de autonomía respecto del Estado, su "secularización" frente a instituciones de regulación social constituidas como la Iglesia, hace de ellas tanto un resultado de dicho movimiento general (donde "modernización" supone emancipación y autonomización de la sociedad civil) como un espacio en cierto punto irreductible a las condiciones generales de dicho movimiento (precisamente por dicha especificidad)

- las creencias respecto a la igualdad en las "condiciones iniciales de lucha" entre los participantes, en la práctica concreta del deporte, por la cual el éxito es el resultado de la calidad y el esfuerzo intrínsecos a la actividad en cuestión, en torno a la igualdad de los actuantes frente a quien ejecuta la justicia deportiva, tanto en el evento como en los tribunales deportivos, la vigencia de un sistemas de reglas escritas (de aplicación primero regional, luego nacional y hoy en día planetaria) cuya responsabilidad está en manos de organizaciones con la misma jurisdicción, constituye un claro índice de su "modernidad" por los atributos que conlleva (burocratización, igualdad "burguesa", meritocracia, democracia formal, etc.); a la vez, la fuerza de este igualitarismo meritocrático universalista debe matizarse por la supervivencia de representaciones en tensión con esos valores, como las que remiten a la importancia de la "grandeza" o la "historia" de algunos competidores (ya sea individuales o grupales) para atribuirles una marca de distinción deportiva o extra-deportiva (caballerosidad, honor, prosapia, etc.) que contradice la antedicha igualdad 
- los lazos entre el desarrollo y expansión planetaria del deporte y el despliegue del sistema mercantil capitalista (y protocapitalista) son notorios; los deportes han sido generados ydesarrollados en sus inicios por dirigentes y emprendedores -así como por profesores, deans, párrocos, etc.pertenecientes o en vinculación con las clases propietarias, y por lo tanto plenamente imbricados en tales relaciones de mercado; sin embargo, desde su nacimiento esta misma cercanía con los lazos y valores mercantiles supuso la elaboración de diferentes respuestas a estas conexiones, siendo la más extendida y conocida la del culto al amateurismo como rechazo elitista a la supuesta o real contaminación mercantil del profesionalismo, a su vez resultante de la confluencia de la novedad deportiva con las relaciones laborales capitalistas en florecimiento

- desde el punto de vista del espacio físico, el desarrollo del deporte también es indisoluble de un rasgo inherente a la "modernidad" como es la urbanización, a tal punto que la ciudades en muchas aproximaciones son sinónimo del mundo moderno, en simétrica equivalencia a la vinculación de "tradicional" con lo rural: los deportes modernos - con la posible excepción del cricket-se desarrollaron en clubes originados y radicados en las cada vez más populosas ciudades, cuyas transformaciones edilicias y urbanísticas (red de transporte, mayor densidad poblacional, tendido de distribución de luz, gas, agua corriente, cloacas, etc.) fueron condición de posibilidad de su existencia; a la vez, el mismo proceso de urbanización hizo peligrar la existencia misma de tales instituciones, al poner los espacios abiertos al interior de las urbes en expansión, indispensables para la práctica deportiva (canchas, campos, baldíos, potreros), en la mira de los emprendimientos inmobiliarios que se dinamizaron fruto del mismo proceso del cual tales prácticas eran resultado y agente.

\section{LA MODERNIDAD LATINOAMERICANA: EL CASO ARGENTINO}

Sustantivo originalmente acotado a un contexto espacial y temporal específico (especificidad que en algunos casos es constitutiva de su definición), ${ }^{11}$ la categoría ha sido aplicada a situaciones históricas diversas, para las cuales la pregunta acerca de su mayor o menor modernidad ha sido una de las vías escogidas para su estudio; para estos casos, el acceso al conjunto de atributos políticos, sociales, económicos y culturales que se asocia con el rótulo constituiría una forma de considerar su inclusión en una dinámica histórica de largo alcance que potencialmente tiende a abarcarlos. Así, la preocupación por su mayor o menor “modernidad” puede apreciarse en el espacio europeo "periférico" a los centros, desde los que habría surgido el despliegue del concepto, al igual que en zonas más alejadas de los mismos.

En nuestra región la pregunta acerca de la modernidad latinoamericana se ha vuelto habitual, a partir del siglo XIX. El grado de alcance del concepto reflejaría, especularmente, la capacidad de resistencia de los obstáculos que impedirían su desarrollo, y cuya supervivencia es vista en muchos casos como incompatible con el mismo. Con particularidades propias de los contextos específicos de referencia y del momento de la producción de estos diagnósticos, los límites de la "modernidad" en América Latina han sido ubicados en la persistencia de modalidades propias del mundo "tradicional": la cultura indígena (e incluso su supervivencia étnica), el peso de las instituciones eclesiásticas, el modo de tenencia y explotación de la tierra, la centralidad de las relaciones económicas precapitalistas, la vigencia de modalidades políticas "patrimonialistas", etc.

De este modo, en el contexto latinoamericano la llegada a la "modernidad" es vista como el cruce de un umbral que es visto al mismo tiempo como inevitable advenimiento de los nuevos tiempos y como alternativa deseable de superación de rémoras que impedirían el desarrollo de sus habitantes. Por tal motivo, el uso de la categoría tendrá una mayor o menor recurrencia de acuerdo al grado con el que este proceso se perciba más o menos problemático, o lo que es lo mismo, de acuerdo a la fortaleza o debilidad que se le asigna al conjunto de obstáculos y dificultades que el universo "tradicional" le oponga al "moderno".

Igualmente, y en un sentido en el que el interés académico aparece teñido de la funcionalidad política del análisis (sin que tal tinte sea las más de las veces intencional, o siquiera evidente, para sus protagonistas), la 
preocupación por detectar las barreras que impidieron o continúan impidiendo el despliegue de los atributos del mundo "moderno" está en la base de los diagnósticos que pretenden guiar la superación de los mismos. La pregunta por la "modernidad" puede en ocasiones deslizarse, por lo tanto, a una propedéutica que dictamine la necesidad de operar una adecuada modernización de las sociedades latinoamericanas, así como los modos de conseguirla.

Desde esta perspectiva, modernidad aparecía como sinónimo y síntesis de algunos rasgos que se veían como definitorios, y cuyo peso específico dependía de las características del momento y el lugar desde donde se realizaba el análisis: así, elementos como el desarrollo económico capitalista, la democracia política liberal, el cosmopolitismo cultural laico, etc., aparecían ante algunos autores tanto como piedra de toque para corroborar el estado de las sociedades locales como en cuanto requisitos a obtener para lograr avanzar en la dirección deseada, al tiempo que las tensiones que guardaban estos rasgos entre sí, y con la realidad local, quedaba más o menos de manifiesto de acuerdo al contexto histórico y social del caso. ${ }^{12}$

Por lo tanto, no es de extrañar la presencia del término en trabajos pertenecientes al campo académico de países como México, Perú, Brasil o Cuba, en los que la importancia de las formaciones sociales anteriores a la conquista europea, junto con la centralidad de estas regiones en la época colonial, se combinaban para producir como efecto la vigencia de elementos reactivos a las transformaciones "modernas", en las múltiples dimensiones anteriormente mencionadas. Paralelamente, la debilidad relativa de las culturas nativas y luego su ubicación periférica en relación a los centros del poder español en América explican la menor utilización de la modernidad como categoría analítica en países como Argentina o Uruguay.

Efectivamente en el caso argentino, la referencia a la modernidad está llamativamente ausente de las principales producciones académicas de los historiadores locales. Por ejemplo, en unautor cuyo objeto y marco de análisis haría esperable la utilización de la categoría como es el caso de José Luis Romero, tanto en sus trabajos sobre el surgimiento de la mentalidad burguesa en la Europa de fines de la Edad Media, como en aquellos centrados en el desarrollo de las ideas en Argentina y más en general en América Latina, en los que la tensión entre el mundo rural y el urbano constituye uno de los factores ideológicos fundamentales, el concepto no es utilizado en ningún momento. Tampoco han hecho uso de la categoría los historiadores que han focalizado su atención en el período que va de mediados del siglo XIX a las primeras décadas del siguiente, es decir, la etapa que en más de un sentido podría considerarse de incorporación de la Argentina a esta "modernidad".

Sin embargo, esta ausencia relativa debe matizarse con algunas excepciones, que pueden agruparse en dos grandes núcleos: por un lado, los trabajos que a partir de los años 50 intentan abordar la cuestión nacional utilizando herramientas de la sociología weberiana, midiendo el grado de modernización relativa de la sociedad argentina; ${ }^{13}$ por el otro, a partir de los años 80 pero con mayor fuerza en los últimos años del siglo XX, el campo de los estudios culturales, donde un conjunto de autores han echado mano de categorías de esta tradición para describir los rasgos de las condiciones de vida urbanas en las grandes urbes argentinas de principios de siglo XX. ${ }^{14}$

En ambos casos el uso de la categoría responde tanto a su funcionalidad como instrumento de conocimiento como a su pretendida eficacia política. En el primer caso, alrededor de la producción desarrollada ejemplarmente por Gino Germani, el análisis de la persistencia de elementos reactivos al desarrollo de la "modernidad" argentina no puede entenderse sin referirlo a la preocupación por comprender al peronismo dentro de ese conjunto de elementos pretéritos cuya insospechada vitalidad hace necesario tanto su análisis como su efectiva remoción: los atributos de una sociedad "moderna" (dominación burocrática, secularización, urbanización, etc.) conviven así, inarmónicamente, con elementos tradicionales que explican y se condensan en el movimiento político encabezado por Perón (ruralismo, migraciones internas, caudillismo carismático, etc.) (Germani, 1987 y 1964). ${ }^{15}$

Por su parte, los trabajos de autores como Beatriz Sarlo recuperando categorías desarrolladas por Raymond Williams (y luego Marshall Berman) para referir a las nuevas sensibilidades urbanas, estéticas, literarias y 
arquitectónicas presentes en la Buenos Aires de los años 20 y 30, no dejan de estar insertos en preocupaciones similares. Aquí la referencia a la "modernidad"de la metrópoli portuaria del período sirve para marcar el contraste con la ruptura posterior de la continuidad del proceso histórico, que encuentra en la violencia política y el terrorismo de Estado de los años 70 su contracara más evidente (Sarlo, 1988; Terán, 1991; Liernur y Silvestri, 1993; Gorelik, 2001).

Así, podría ser interesante pensar que en Argentina, si la producción académica de los años 50 está ligada al "desarrollismo" como modelo económico y político de articulación entre el análisis de las condiciones sociales y su necesaria transformación, en la década de 1980 la cultura política de la "recuperación de la democracia" se tradujo en una tematización de la modernidad desde la perspectiva de una "cultura democrática", contracara de la violencia y la intolerancia políticas que habrían imperado en el país a partir de cierta fecha. Si en un caso la "modernidad" es un objetivo a alcanzar, en el segundo se trataría más bien de una etapa perdida, pérdida a la que el contraste con la experiencia inmediata de los años del terrorismo de Estado no hace más que volver aún más amarga.

\section{DEPORTE: ¿UNA “MODERNIZACIÓN” ARgENTINA?}

Después de esta extensa revisión general de las tensiones derivadas de la presencia de la categoría en los abordajes sobre las transformaciones atravesadas por la región en los últimos siglos, pasemos a interrogarnos acerca de los problemas que conlleva su utilización para el caso concreto que aquí nos ocupa: la llegada, despliegue y finalmente adopción delas prácticas deportivas "modernas" por la sociedad argentina durante un período bien delimitado de nuestra historia reciente. ${ }^{16}$ En este trabajo nos hacemos una pregunta de la que, adelantamos,no sabemos la respuesta: ¿fueron las prácticas deportivas el resultado de esos cambios más generales a los que se suele aludir con la referencia a la "modernización" de la sociedad argentina, o fue, inversamente, el mismo deporte el agente de tales cambios? Esta pregunta, evidentemente, se relaciona con otras: ¿cuáles fueron los elementos novedosos que aportaron los deportes? ¿En qué sentido se relacionan con las nuevas formas de vida colectiva?

Una traducción más acotada y al mismo tiempo más sugerente de esas inquietudes generales podría ser la siguiente: ¿cómo podemos pensar la incorporación de ideas, valores, códigos de comportamiento, "ethos", ligados a la vez a las coordenadas del universo "burgués" (es decir, "moderno") y "tradicional" (o "caballeresco"), en sociedades como la argentina o más en general las de las flamantes repúblicas independientes de América Latina, carentes de un pasado que corresponda estrictamente a los parámetros de lo que en el caso europeo era el mundo "medieval"? La paradoja de la llegada y posterior popularización de las prácticas deportivas británicas en espacios culturales y sociales como el del Buenos Aires de la segunda mitad del XIX estriba precisamente en que con ellas venían implícitos tanto valores y mentalidades "modernas", propias del capitalismo en expansión, como así también otras mucho más antiguas, arrastradas en sus lugares de origen de un pasado que no había tenido lugar en América. Exagerando con afán de ilustrar mejor el punto, podríamos aventurar que con el deporte (como con tantas otras formas de conducta adoptadas en el período) lo que llega a nuestros espacios culturales es tanto el moderno capitalismo como el antiguo y disuelto mundo medieval.

Para avanzar en la elucidación de estos interrogantes debemos echar mano de algunas de las múltiples y en cierto punto contradictorias aristas que, como vimos, presenta nuestra ubicua y problemática categoría. Efectivamente, si observamos la plasmación de los atributos generalmente atribuidos a la "modernidad"en sus múltiples facetas: económicas, políticas, culturales, actitudinales, o genéricamente "sociales"-en los momentos iniciales de la llegada del deporte a la Argentina, en especial a la ciudad de Buenos Aires, y su despliegue entre grupos sociales, reconocemos sin dificultad que este proceso ha tenido similitudes con los procesos vividos en buena parte del planeta fruto del contacto del capital inglés y sus gerenciadores y empleados en diversas regiones, particularmente en nuestra región (Arbena, 1988). 
El tema merece algún detenimiento debido a que ese papel lo cumplieron los británicos, con sus peculiaridades. Por ejemplo, sus concepciones acerca de la misión civilizatoria del deporte eran claras, y sin embargo, esa iniciativa se desplegó de manera paradójica ya que no fueron muy permeables a que otros grupos sociales participaran de sus emprendimientos deportivos ni de sus clubes. De hecho, el énfasis en preservar la exclusividad británica en sus clubes indica hasta qué punto hacían de la preservación de esas diferencias un valor en sí mismo. Por el contario, los nuevos clubes, surgidos por la acción militante y entusiasta de sus jóvenes fundadores, pertenecientes a los sectores medios y medios-bajos de la sociedad "criolla", focalizaron su atención en la apertura de sus puertas, debido a la necesaria convocatoria a una mayor cantidad de miembros que pagaran una precaria cuota social; así, un atributo intrínseco de las pautas "modernas" de comportamiento y ordenamiento colectivo, como puede ser el igualitarismo formal de los miembros de una asociación civil, aparece de la mano de las entidades vinculadas a los sectores que en principio serían más reacios a tales parámetros, mientras que las instituciones que simbolizarían por antonomasia tales rasgos aparecen por el contrario imbuidas de un espíritu jerarquizante y estamental más propio, en principio, de la tradición a la que vendrían a desalojar.

En este sentido, la llegada y difusión del formato de clubes en Buenos Aires no fue similar, ni mucho menos copia carbónica, del ejemplo inglés. El "asociacionismo"-es decir, la tendencia de distintos sectores de la vida porteña no sólo a reunirse en espacios más o menos horizontales para defender intereses comunes o vehiculizar actividades diversas para beneficio de sus miembros, sino sobre todo a plasmar tales agrupamientos en instituciones formales, dotadas del aparato burocrático y administrativo y con la firme pretensión de perdurar en el tiempo-, fue horizonte común de época, compartido desde empleados británicos del ferrocarril hasta socialistas y comunistas: se lo encuentra tanto en el afán de los jóvenes de los incipientes barrios porteños por fundar clubes de fútbol como en los sindicatos y asociaciones de fomento de obreros, empleados y vecinos, o en los partidos políticos y cámaras y corporaciones de los sectores profesionales más acomodados. Se trata de iniciativas comunes muy "modernas", en cierto sentido, pero que miradas con cierto detenimiento se ve que recubren en cada caso lógicas muy disímiles. Lo relevante aquí es de qué modo estos ejemplos permiten observar las dificultades de la adecuación de las categorías modelizadoras como modernidad a la mucho más matizada riqueza de los resultados empíricos de nuestras investigaciones; "modernidad” y "tradición” parecen aquí ir más de la mano que en la contradicción inherente a su definición canónica (Frydenberg, 2011).

Desde luego, se nos podría objetar que estas ambigüedades no son tanto propias del concepto en sí, sino de la forma peculiar de desarrollo alcanzada por la sociedad británica en los siglos precedentes, con grupos sociales cuyos comportamientos, valores y actitudes ante la vida resultaban de una mixtura entre formas caballerescas, acumulación protocapitalista, ethos protestante ("moderno"), radicalismo político y social milenarista, y tantos otros etcéteras. Sin entrar en la discusión sobre tal punto, y sin negar las particularidades típicas de la historia de la sociedad británica, lo que queremos señalar aquí es sencillamente que tales rasgos idiosincráticos son en algún punto típicos de las dificultades de cualquier formación político-cultural a la hora de pasar el análisis de su desarrollo histórico por la criba de categorías sustantivadas como la que aquí nos atañe. En esta como en tantas otras ocasiones vale la máxima de Goethe que opone la verde vitalidad de la vida (histórica, en nuestro caso) a la grisácea y monocromática coloración de los modelos teóricos.

En lo que sigue quisiéramos mostrar algunos ejemplos de las dificultades que conlleva la aplicación acrítica de un instrumento como la categoría modernidad para la comprensión acabada de las transformaciones históricas verificadas en el proceso de difusión y popularización de las prácticas deportivas de origen británico en Buenos Aires. La breve enumeración (en ningún modo exhaustiva) resulta de la experiencia directa de trabajos de investigación desarrollados en el pasado.

Al hablar de posibles efectos modernizantes del implante del deporte en nuestra sociedad, nos referimos al instante inicial, es decir, la etapa "británica", desde mediados del siglo XIX hasta la segunda década del siglo XX. Un primer problema es el de los potenciales elementos de tensión que existen entre el "modelo 
británico" que representaría el sporty la reconocida influencia francesa en los sectores sociales dominantes; ¿de qué modo se articularon ambas influencias en el comportamiento concreto de la "elite" porteña? ${ }^{17}$

Este tema no es menor, ya que muchos trabajos han mostrado el carácter problemático del desarrollo del deporte en el continente europeo, y en particular en Francia, donde la influencia inglesa fue recibida con una mezcla de admiración y recelo (Gounot, Jallat y Caritey, 2007). Sin pretender adscribir el comportamiento de la clase dominante argentina a la de su equivalente francesa, no sería conveniente dejar de lado este aspecto de la cuestión, a riesgo de perder de vista los elementos diversos que confluyenen las influencias culturales "modernizadoras" que terminan expresándose en la adopción de determinadas prácticas.

Por otro lado, si desde los sectores altos el tema de la "modernización" por el deporte presenta aristas problemáticas, tampoco es lineal el panorama desde la perspectiva simétrica. En efecto, ¿qué podemos decir de esas prácticas y sus valores concomitantes cuando son adoptadas por los sectores populares(entendiendo por tales a los grupos mayoritarios, definidos por la negativa: los no-británicos, no-elite)? El punto es delicado, ya quela popularización de las prácticas implica un cambio en las mismas, y particularmente en cuanto a los "valores" asociados, por lo que la pregunta sobre la "modernidad" del deporte en esta etapa no es ociosa.

Un ejemplo ilustrativo: la popularización de la práctica del fútbol en Buenos Aires hizo perceptible una modificación en el sistema de valores en algunos aspectos centrales; el fairplay pasa a ocupar un rango menor (pero sin extinguirse), mientras que el "éxito" emerge como bien supremo; del mismo modo, entra en discusión la "objetividad" y la "justicia”, tanto la impartida por el árbitro como la referida al resultado deportivo, todos los cuales pasan a ser objeto de debate; en consecuencia, se desarrollan estratagemas discursivaspara intentar evitar las consecuencias de la derrota (Frydenberg, 1997).

¿Son estos valores "populares" más o menos "modernos" que los que están asociados al sport que practicaban los miembros de los exclusivos clubes y colegios de la comunidad británica? Es un tema abierto, pero sirve para indicar las complejidades del proceso de incorporación de estas nuevas prácticas "modernas", así como los múltiples sentidos que puede tener el término de acuerdo al objeto empírico sobre el que se lo aplique.

Por otro lado, es imposible hablar de novedades introducidas por el deporte sin hacer mención a los cambios urbanos. Como se mencionó, el desarrollo mismo de las ciudades es considerado como sinónimo y condición de posibilidad de la modernidad; en nuestro caso, el fútbol ha crecido con el desarrollo de la ciudad sobre la pampa. No obstante, este mismo desarrollo compite con otras expresiones conspicuas del crecimiento urbano: la propiedad privada del espacio, y también el área estatal, que va diseñando calles, plazas, hospitales, escuelas, y que en muchos casos desalojaron a los jóvenes deportistas de lo que ellos pretendían fuera su cancha.

La misma "modernidad", entonces, se manifestaría tanto en la adopción de las prácticas deportivas, como en la modificación de las pautas de sociabilidad de las personas, y en las formas de apropiación del espacio urbano; referir estos desarrollos a manifestaciones de un mismo proceso no hace más que ocultar la tensión que se oculta detrás de ellos, escondiéndolos detrás de una aparente uniformidad transformadora.

Los jóvenes de los sectores populares, en su afán por crear equipos-clubes que contaran con terrenos donde practicar fútbol, se encontraron con enormes dificultades.Losclubes que pudieron sobrevivir pusieron buena parte de sus energías en sostener el espacio que habían logrado conseguir con el objetivo de edificar sus estadios. Todos fueron emprendimientos de los propios clubes; no hubo en Buenos Aires un estadio que dependiera de las finanzas municipales o nacionales. Es decir, el deporte acompañó, pero compitió fuertemente con el desarrollo de la llamada ciudad moderna. El deporte promovido por los sectores populares debió edificarse sobre la base de su propia iniciativa. El deporte promovido por los integrantes de la colonia inglesa se afincó en las afueras de la ciudad. ${ }^{18}$

Este tema nos lleva al de la relación entre el desarrollo del deporte con el aparato estatal. Desde los años 1860 se estaba creando el Estado-nación con un texto constitucional, una elite en construcción y la apertura comercial dominando la economía nacional dentro del modelo agroexportador. La instalación de la práctica 
deportiva coincidió con este marco general, conservador en lo político, liberal enlo económico. Sin embargo, muchas de las entidades deportivas vivieron experiencias internas con mayor grado de gimnasia democrática que la existente en la vida política nacional.

En este sentido puede decirse que algunas de las entidades deportivas se adelantaron a las novedades que se impusieron (muy limitadas y formales) desde 1916 con el sufragio universal. Sin embargo, los clubes no estuvieron exentos de las viejas maneras de combinación del quehacer político,entre las prácticas horizontales democráticas y el paternalismo de notables y caudillos.

\section{LA MODERNIDAD COMO “MODELO": CONTINUIDADES Y RUPTURAS}

Los ejemplos anteriores no hacen más que ilustrar un problema inherente al trabajo del historiador: la tensión que existe entre la necesaria utilización de categorías ("modelos") para, a través de ellas, comprender conceptualmente la realidad histórica empíricamente registrada, y la posibilidad de que tales herramientas obstaculicen el registro de los detalles fácticos menos armónicos con ellas. Si este problema es característico del campo, en este caso se ve agravado por la particular plasticidad de la "modernidad" como instrumento analítico.

Sin embargo, las posibilidades que abre la categoría son innegables. Por empezar, debido al hecho de que los propios protagonistas del período utilizan el concepto de "modernidad" para pensar los cambios históricos experimentados y para pensarse a sí mismos en dichos cambios. Innumerables testimonios demuestran cómo los primeros deportistas, tanto ingleses como de la elite criolla y los sectores populares, por el hecho mismo de ser deportistas, se sumaban al progreso y a los atributos de la civilización, y experimentaban participar de novedades en el sentido progresivo, civilizatorio e higienista. Esta "modernidad" era naturalmente tributaria de una tarea permanente de construcción de la opinión pública a través de medios de comunicación e instituciones estatales y de la propia sociedad civil; es a partir de esta insistente reiteración que quizás pueda explicarse la apariencia y visibilidad de la palabra y la creencia en la "modernidad" de los cambios experimentados. A pesar de esta evidente utilidad de la categoría, estamos obligados a avanzar más allá de su uso en la "voz nativa" de los actores, si suponemos que la posesión de categorías propias de la perspectiva del observador forma parte de las condiciones básicas de la tarea del investigador. Sin entrar aquí en la antigua discusión entre las categorías antropológicas emic y etic, digamos sólo que nuestro punto de vista es que la posición del historiador le exige echar mano de categorías no sólo distintasa las de sus analizados, sino muchas veces en contradicción con ellas.

En el caso del deporte, que es el que nos ocupa aquí, podría suponerse que estas prácticas, a pesar de su evidente "novedad"-visible tanto en sus aspectos más superficiales y materiales (como las características técnicas de los implementos que los nuevos juegos requieren), como en aquellos más abstractos y profundos (como las nuevas formas de ejercer y tolerar la violencia física propia y ajena, la actitud ante la victoria y ante la derrota, las formas de relación con las normas y la autoridad encargada de aplicarlas, etc.)-, incluyen elementos de vieja data, que emergen en la práctica deportiva y en el ritual del espectáculo. ¿Cuáles pueden ser esas conexiones que relacionen el deporte actual con fenómenos añejos y por lo tanto pre-"modernos"?

Se suele hacer hincapié en los costados que asocian el deporte al desarrollo del individualismo emergente en la sociedad medieval a partir delos siglos X-XI. En el otro extremo de este arco se ubicarían las relaciones comunitarias hegemónicas en esa sociedad, que paradójicamente podrían tener un eco en nuestros deportes en la construcción de las identidades colectivas mediante la participación en el ritual del espectáculo. Otra franja con connotaciones con un pasado lejano son los valores adheridos de manera más o menos cercana al código ético del fairplay, vigente en el momento de la génesis del deporte traído con él a nuestras tierras. Tal código, si bien contiene valores y creencias asociadas a la burguesía, tieneraíces en la ética nobiliaria y caballeresca. El gentleman, deportista por antonomasia, era un hibrido que congeniaba valores aristocráticos tradicionales con actitudes y creencias burguesas muy modernas. 
Como hemos indicado más arriba, existe una tensión desde sus inicios entre la nueva "tradición" amateurista junto con la desafiante profesionalización, tensión que en nuestro continente se manifiesta en la contraposición entre las influencias "modernizadoras" del deporte de origen británico y las del proveniente de Estados Unidos. Por otro lado, no podemos obviar que el honor fue centro alrededor del cual se gestionaron actitudes de las clases dominantes de otros sistemas sociales, y con sus grandes modificaciones fue y sigue siendo en parte el norte que orienta algunas de las actitudes de nuestros hinchas.

Si bien fue tarea emprendida por la sociedad civil, muchos de los contornos de lo que hoy conocemos como deporte fueron fijados por los conflictos ocurridos con las emergentes formas de estatalidad desde el siglo $\mathrm{XIV} \mathrm{y} \mathrm{XV,} \mathrm{los} \mathrm{nacientes} \mathrm{estados} \mathrm{monárquicos.} \mathrm{Esto} \mathrm{es} \mathrm{así} \mathrm{porque,} \mathrm{desde} \mathrm{luego,} \mathrm{los} \mathrm{rasgos} \mathrm{de} \mathrm{los} \mathrm{agrupamientos}$ sociales (y por supuesto, los de los individuos que viven en ellos) se ven irremediablemente modificados por el desarrollo y modificación de las formas políticas de los mismos. ${ }^{19}$

El espejismo teleológico inherente en categorías como modernidad o modernización nos hace buscar hacia atrás los elementos que suponemos se plasmarán en el futuro. Sin embargo, en muchas oportunidades la historia nos ha mostrado la vitalidad de fenómenos que considerábamos muertos, por pertenecer a formaciones social-culturales que estaban "en vías de desaparición" siguiendo patrones de sucesión entre etapas que operan de modo similar al que subyace detrás de categorías como modernización. Por tal motivo, la paradójica propuesta que esbozamos, entonces, sería pensar a las prácticas deportivas como, efectivamente, cruzadas por procesos y transformaciones que suponen una ruptura indiscutible con formas precedentes de comportamiento, pero a la vez, manteniendo vivas en su interior un conjunto de prácticas y valores que pertenecen a universos mucho más antiguos a los de la "modernidad"; el deporte sería así el resultado de múltiples y cruzadas influencias y procesos;algunos, efectivamente, ligados indisolublemente al ethos capitalista o a la "modernidad" en general, pero muchos otros de una antigüedad que puede remontarse a decenas de siglos en el pasado, muchos de los cuales están en tensión manifiesta con los anteriores.

\section{BibLiOgRAFìA}

Anderson, P. (1984).Modernity and Revolution.NLR, 144.

Appadurai, A. (2001). Modernidad desbordada. Dimensiones culturales de la globalización. Montevideo:Trilce [1996]. Arbena, J. (1988).Sport and Society in Latin America: Diffusion, Dependency and the Rise of Mass Culture. Westport: Greenwood Press.

Archetti, E. (2003).Masculinidades: fútbol, tango y polo en la Argentina. Buenos Aires: Antropofagia [1999].

Berman, M. (1989).Todo lo sólido se desvanece en el aire. La experiencia de la modernidad.Madrid: Siglo XXI.

Blanco, A. (2006).Razón y Modernidad. Gino Germani y la Sociología en la Argentina. Buenos Aires: Siglo XXI.

Blumenberg, H. (2008).La legitimación de la Edad Moderna. Valencia: Pre-Textos [1966].

Bourdieu, P. (1993).Programa para una sociología del deporte.EnCosas dichas. Barcelona:Gedisa.

Calinescu, M. (1991).Cinco caras de la modernidad. Madrid:Tecnos[1977].

Carrington, B. (2010). Race, Sport and Politics: The Sporting Black Diaspora.Londes: SAGE.

Cohen, J.,Hazelrigg, L. y Pope, W. (1975).De-Parsonizing Weber: A Critique of Parsons' Interpretation of Weber's Sociology.American Sociological Review, 40(2).

Eisenstadt, S.N. (2003).Comparativecivilizations and multiplemodernities. Leiden: Brill

Elias, N. (1994).El proceso civilizatorio.México:FCE[1939].

Elias, N. y Dunning, E. (1995).Deporte y ocio en el proceso de la civilización. México:FCE [1986].

Foucault, M. (2002).¿Qué es la ilustración?Córdoba: Alción [1984].

Frydenberg, J. (1997).Prácticas y valores en el proceso de popularización del fútbol. Buenos Aires, 1900-1910.Entrepasados. Revista de historia, 6(12). 
Frydenberg, J. (2011).Historia social del fútbol: del amateurismo a la profesionalización. Buenos Aires: Siglo XXI.

García Canclini, N. (2001).Culturas híbridas. Estrategias para entrar y salir de la modernidad. Buenos Aires: Paidós.

Germani, G. (1987).Estructura social de la Argentina. Buenos Aires: Solar-Hachette [1955].

Germani, G. (1964).Politica y sociedad en una época en transición. Buenos Aires: Paidós.

Giddens, A. (1999).Consecuencias de la modernidad. Madrid: Alianza [1990].

Giddens, A. (1995). Modernidad e identidad del yo. Barcelona: Península [1991].

Gorelik, A. (2001).La grilla y el parque. Buenos Aires: UNQ.

Gounot, A., Jallat, D. y Caritey,B. (comps.). (2007).Les Politiques au stade. ÉtudecomparéedesmanifestationssportivesduXIXeauXXesiècle. Rennes:PressesUniversitaires de Rennes.

Guttmann, A. (1978).From Ritual to Record: The Nature of Modern Sports. Nueva York: Columbia UP.

Habermas, J. (1998).La modernidad: un proyecto inacabado.Punto de Vista, 21 [1980].

Habermas, J. (1989). El discurso filosófico de la modernidad. Madrid: Taurus [1985].

Horkheimer, M. y Adorno, T. (1998).Dialéctica de la Ilustración.Madrid:Trotta[1969].

Jameson, F. (2004).Una modernidad singular. Barcelona:Gedisa [2002]

Koselleck, R.(1993).Futuro pasado. Barcelona: Paidós [1985].

Latour, B. (2007).Nunca fuimos modernos. Buenos Aires: Siglo XXI.

Liernur, F. y Silvestri, G. (1993).El umbral de la metrópolis: transformaciones técnicas y cultura en la modernización de Buenos Aires, 1870-1930. Buenos Aires: Sudamericana.

Löwith, K. (1956).El sentido de la historia. Implicaciones teológicas de la filosofía de la historia. Madrid: Aguilar [1949].

Osborne, P. (1992).Modernity is a Qualitative, not a Chronological, Category.NLR, 192.

Palti, E. (2005).La modernidad como problema. El esquema 'de la tradición a la modernidad' y la dislocación de los modelos teleológicos.Modernidades, 1, Universidad de Córdoba.

Rosa, H.(2005). Social Aceleration. A New Theory of Modernity. Nueva York: Columbia UP [2004].

Sarlo, B. (1988).Una modernidad periférica: Buenos Aires 1920 y 1930. Buenos Aires: Nueva Visión.

Terán, O. (1991).Nuestros años sesentas. Buenos Aires:Puntosur.

Therborn, G. (1992).Peripecias de la modernidad. Buenos Aires: El Cielo por Asalto.

Therborn, G. (1996).Dialectics of Modernity.NLR,215.

Therborn, G. (2003).Entangled Modernities.European Journal of Social Theory, 6(3).

Touraine, A. (1993).Crítica de la modernidad. México: FCE.

Turner, V. (1988).El proceso ritual. Estructura y antiestructura. Madrid: Taurus [1969].

Wagner, P. (1994).A Sociology of Modernity. Liberty and Discipline.Londres:Routledge.

Weber, M. (1997).Acerca de laobjetividad cognoscitiva en ciencia social y en política social.EnEnsayos sobre metodología sociológica. Buenos Aires:Amorrortu [1904].

Williams, R. (2001).El campo y la ciudad. Buenos Aires: Paidós [1977].

Williams, R (2003).Palabras clave. Buenos Aires: Nueva Visión [1976].

Xavier-Guerra, F. (1992).Modernidad e independencias.Madrid: Mapfre.

\section{Notas}

1 Las discusiones acerca del concepto son casi tan variadas como sus usos. A modo de ejemplo: la crítica de Anderson a Berman (Anderson, 1984), y la respuesta de este último; Osborne,1992; Therborn (1996 y 2003), Calinescu, 1991; Jameson, 2004, Habermas 1998 y 1989. Cf. también el debate entre Löwith y Blumenberg sobre la modernidad como escatología religiosa secularizada (Löwith, 1956 y Blumenberg, 2008). También Raymond Williams ha reflexionado sobre el concepto (Williams, 2003), aunque a partir del genérico Moderno, y Michel Foucault se ocupó de definir la “actitud” de la modernidad en su trabajo sobre el seminal “¿Qué es la ilustración?” de Kant (Foucault, 2002). 
2 Sobre el "abuso" del término. cf. Jameson, 2004.

3 Sobre el teleologismo de la relación "modernidad"/"modernización" cf. los trabajos de los estudios postcoloniales de autores como Homi K. Bhabha o GayatriSpivak.

4 Sobre la crítica al sentido unívoco del término para dar cuenta de las transformaciones ocurridas en el contexto hispanoamericano, Xavier-Guerra (1992) y Palti (2005).

5 Georg Simmel es particularmente atento a la degradación del mundo del "espíritu" frente a la "cosificación" característica de la "cultura objetiva"; desde luego, tal "desrealización" o "inautenticidad" está en el centro de las preocupaciones de la Escuela de Frankfurt, como en Horkheimer y Adorno (1998), así como las de Walter Benjamin y los ya mencionados Berman y Habermas.

6 Los trabajos dentro de esta línea generalmente se ubican en la estela dejada por las obras de Marx y Weber (y en menor medida de Simmel, en cuanto a la monetarización de la economía).

7 Sólo para referirnos a los autores pertenecientes ala sociología clásica, citemos la oposición entre el modo de producción feudal y capitalista en Marx, entre Gemeinschaft y Gessellschaft en Tönnies, o entre solidaridad mecánica y orgánica en Durkheim, y sobre todo entre forma de vida "tradicional" y "moderna" (y el despliegue de la racionalidad "con arreglo a fines") en Weber. También mencionemos los trabajos de Simmel (en cuanto a la relación entre "vida" y "formas" en el mundo moderno) y de Elias (sobre la convergencia entre el desarrollo de las formas estatales y el de los mecanismos de autocoacción individual). Recientemente, ha sido Giddens quien más ha hecho uso del término (1993 y 1995).

8 La referencia aquí es sobre todo Williams (2001), haciendo uso de categorías nacidas de la influencia de Antonio Gramsci.

9 La categoría zonas libres a la que Archetti adscribe las prácticas deportivas proviene de la antropología simbólica de Turner (1988). Desde otra dirección, cf. Bourdieu (1993)y Elias y Dunning (1995).

10 Para una síntesis de los rasgos de la versión parsoniana de Weber (basada en la interpretación alternativa proporcionada por la obra de ReinhardBendix), cf. Cohen, Hazelrigg y Pope, 1975.

11 Therborn(2003) señala el eurocentrismo "cándido" de Giddens cuando define modernidad como "modalidades de vida o de organización sociales que emergieron en Europa a partir de aproximadamente el siglo XVII, y que luego adquirieron influencia más o menos mundial”.

12 El interés por la "modernidad", en este sentido, es característico de los enfoques desarrollados en diversas disciplinas del campo de las ciencias sociales en los años 50 y 60, dentro del contexto mundial originado en los años posteriores a la II Guerra Mundial; cf. Wagner, 1994.

13 Vinculados a los enfoques desarrollados en diversas disciplinas sociales en el contexto mundial posterior a la II Guerra Mundial; cf. Wagner, 1994.

14 En los últimos años ha aparecido la publicación electrónica Modernidades (Universidad de Córdoba, Facultad de Filosofía y Humanidades, actualmente interrumpida), lo que matiza esta afirmación, aunque no deja de ser significativo que sea Córdoba la sede de un proyecto de este tipo, habida cuenta de su mayor importancia en el espacio colonial del Virreinato.

15 Sobre Germani, cf. Blanco, 2006.

16 En lo que sigue nos basaremos en los resultados de distintas investigaciones anteriores, algunas de las cuales aparecen volcadas en Frydenberg, 2011.

17 Sobre las relaciones culturales entre Francia y nuestro país, cf., entre muchos otros, los trabajos de Hebe Pelosi y de Hernán Otero.

18 Las pocas instalaciones deportivas edificadas por la elite dominante se emplazaron en zonas parquizadas no edificadas de la ciudad (como GEBA o la Sociedad Sportiva).

19 Cf. Elias(1994) sobre el paralelismo entre la "psicogénesis" y la "sociogénesis" de la civilización. 